\title{
ODF : la classe I canine est-elle toujours pertinente?
}

\section{RÉSUMÉ}

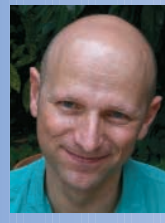

\section{Frédéric HAÏM}

Chirurgien-dentiste,

Spécialiste qualifié

en Orthopédie dento-faciale,

11 , rue de la convention,

75015 Paris.
La recherche d'une classe I canine s'est imposée comme un objectif prioritaire des traitements orthodontiques. Certaines études, commanditées par des organismes de protection sociale, ont pu conclure que son absence en fin de traitement était un critère de non qualité de ce traitement.

Cependant, dans certaines situations cliniques, une classe I canine ne pourrait être obtenue qu'au détriment d'autres objectifs non moins impérieux.

Un classement des priorités dans une approche résolument interdisciplinaire est indispensable. Face à la diversité des situations cliniques, les limites et les dangers d'une standardisation abusive sont évoqués.

L'écoute du patient dans le cadre du colloque singulier, l'échange interdisciplinaire, la réflexion, la liberté thérapeutique et l'indépendance professionnelle au service de l'éthique, sont les gages d'une médecine de qualité au service du patient. 


\section{Introduction}

$>$

Cette interrogation, volontairement

provocatrice, s'inscrit dans une démarche scientifique qui impose la remise en cause régulière des connaissances supposées acquises, pour en éprouver la solidité.

C'est à ce prix que les piliers ressortent renforcés, ou au contraire, doivent être modifiés, que les modèles sont perfectionnés, et que la connaissance progresse loin de tout dogmatisme et sans préjugé.

Définie par Angle [1] en 1907, I'occlusion engrenante de classe I a été décrite comme la relation normale ou optimale de l'occlusion dentaire humaine.
Pour I'ANAES, (Agence Nationale d'Accréditation et d'Évaluation en Santé) la classe I canine fait partie des critères d'aboutissement des traitements orthodontiques [2]. Des études récentes menées sous l'égide de I'URCAM (Union régionale des caisses d'assurance maladie) retiennent son absence, comme un critère de non qualité d'un traitement [3].

Après un bref rappel des avantages procurés par des relations de classe I, tant sur le plan statique, dynamique que cinématique, un certain nombre de situations cliniques seront évoquées pour lesquelles l'obtention d'une classe I canine est, soit impossible, soit en contradiction avec d'autres impératifs thérapeutiques [4].

\section{Les avantages de l'occlusion de classe I}

Nous préférons parler des avantages procurés par des rapports de classe I, plutôt que de normalité de la classe I.

Il existe souvent une confusion entre norme, normalité et moyenne statistique. Nous devons garder à l'esprit que la notion de normalité statistique est peu respectueuse de la diversité biologique et ne tient compte ni des capacités d'adaptation d'un individu, ni des différentes manières dont une fonction harmonieuse peut s'accomplir.

Ces réserves étant faites, nous apprécions la classe I car elle procure :

- sur le plan statique, un engrènement qui stabilise les dents par des contacts cuspides/ fosses ou cuspides/embrasures ;

- sur le plan dynamique, des forces équilibrées et parallèles au grand axe des dents des secteurs latéraux ;

- sur le plan cinématique: le guidage de la mandibule dans les mouvements de latéralité par des dents dont la racine est robuste et dont l'abondance de capteurs desmodontaux renseigne les centres moteurs sur l'intensité des forces et la direction des mouvements. En outre, elle assure la présence de structures d'échappement des cuspides d'appui - embrasures et fosses - de morphologies adaptées et orientées dans le sens du mouvement.

La mise en place d'une classe I canine et d'une protection canine constitue pour l'orthodontiste la manière la plus simple de provoquer une désocclusion des secteurs postérieurs en latéralité et de prévenir le risque d'interférence postérieure du côté non travaillant. Ces interférences semblent être, avec la morsure directe sur le parodonte, dans les cas de supraclusion sévère et la prématurité responsable d'un latéroglissement mandibulaire, la disposition la plus potentiellement pathogène des malocclusions $[5,6,7]$. 


\title{
Critique de la valeur prédominante de la classe I
}

\author{
et du concept de protecton canine \\ en denture naturelle
}

Sans être véritablement remise en cause, c'est la prédominance de ce facteur qui a été critiquée.

Si la protection canine constitue une manière simple d'éviter des interférences du côté non travaillant, la "fonction de groupe», caractérisée par des contacts équilibrés et bien répartis en latéralité du côté travaillant est parfaitement fonctionnelle, bien que plus délicate à obtenir pour l'orthodontiste.

Tout un courant de pensée orthodontique dit "de la réhabilitation neuro occlusale», inspiré par les travaux de Planas [8], défend I'idée d'une occlusion de type balançant (traditionnellement utilisée en prothèse adjointe) avec des contacts du côté travaillant et non travaillant. Une attention particulière est portée sur les guidages en latéralité pour qu'ils pro- voquent un minimum d'abaissement mandibulaire avec le même degré d'abaissement à droite et à gauche.

Pour les tenants de cette philosophie orthodontique, la classe I canine n'est pas un critère essentiel et a contrario, une protection canine "trop forte» avec une pente canine trop abrupte provoquant un grand abaissement mandibulaire, serait susceptible de compromettre la santé du système stomatognatique en réduisant les possibilités d'excursion mandibulaire. Elle serait responsable du faible développement transversal des arcades dentaires et sagittal de la mandibule.

Pour illustrer notre propos, des situations rendant impossible ou discutable l'obtention d'une classe I canine seront décrites, sans prétendre à l'exhaustivité.

\section{La morphologie inadaptée des canines}

Des canines particulièrement fines et acérées ne permettront pas le guidage canin en relation de classe I.

En latéralité, le versant distal de la canine mandibulaire frôle le versant mésial de la canine maxillaire sans établir de contact. Les deux versants présentent une concavité au lieu de la convexité habituelle (fig. 1).

Cette anomalie de forme peut être compensée par une adjonction de composite ou plus sûrement par la recherche d'une classe II canine modérée qui rendra le guidage fonctionnel.

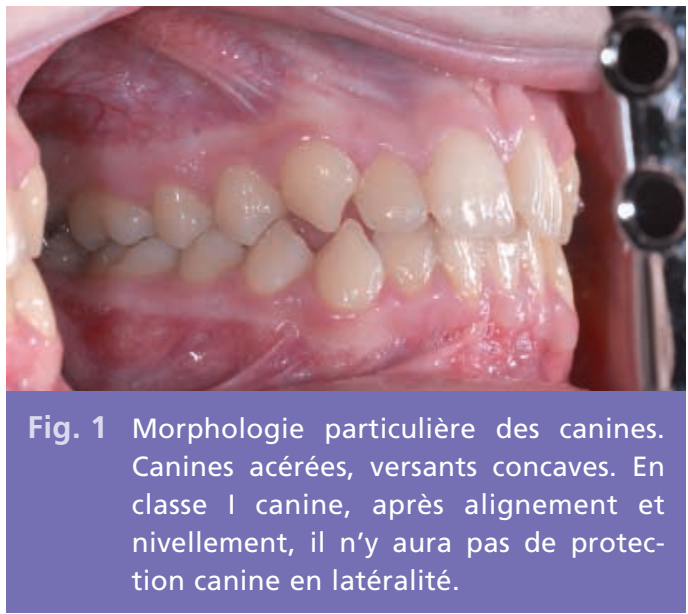




\section{La dysharmonie dento-dentaire}

La plus fréquente et la mieux étudiée, est la dysharmonie dento-dentaire par excès mandibulaire ou par défaut maxillaire, fréquemment liée à la réduction du diamètre des incisives latérales maxillaires. Elle est calculée par I'indice de Bolton [9, 10] et a été souvent sous-estimée dans le diagnostic orthodontique.

Elle impose un compromis entre trois options :

- une classe I canine avec la persistance de diastèmes maxillaires (fig. 2 a, b) ;

- une classe II canine modérée, sans diastème (fig. 3 a, b) ;

- une réduction amélaire des dents mandibulaires.
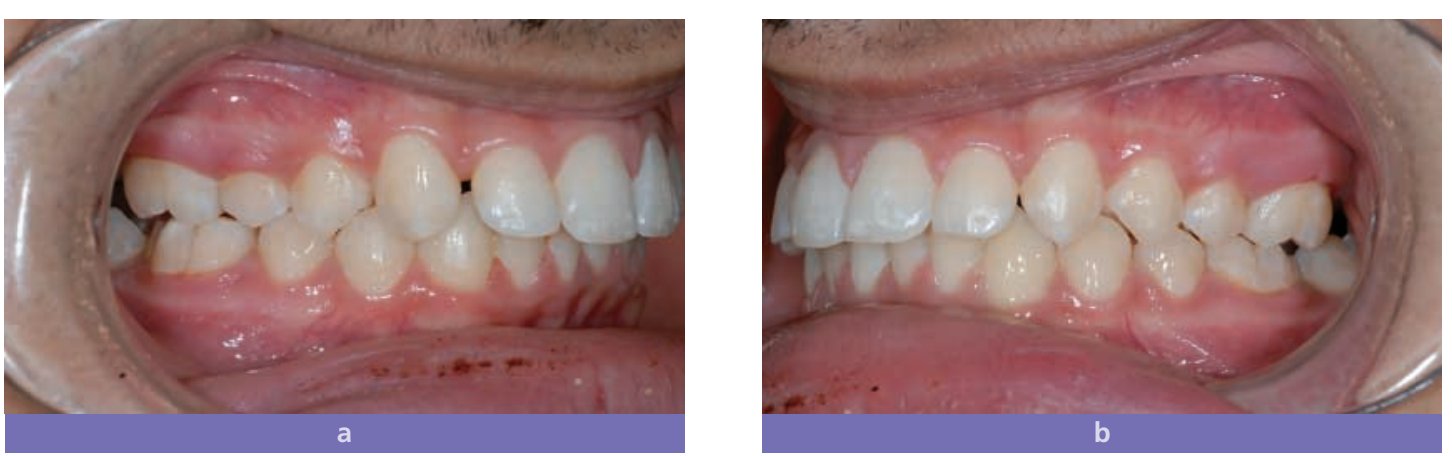

Fig. 2 a et $\mathbf{b}$ Dysharmonie dento-dentaire : classe I canine avec persistance d'un diastème 12-13 malgré un surplomb et un recouvrement normal.
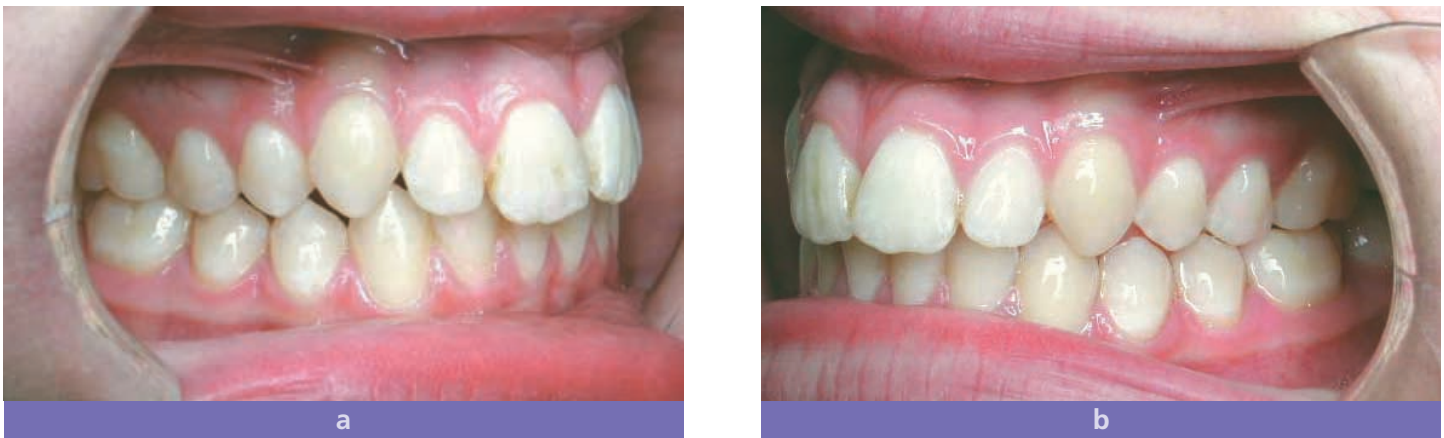

Fig. 3 a et b Dysharmonie dento-dentaire. Classe I molaire, classe II canine.

La dysharmonie des secteurs postérieurs, encore plus négligée, vient de faire l'objet d'une publication récente [11]. Elle est responsable de la difficulté d'obtenir concomitamment, une classe I canine et une classe I molaire (fig. 4 a, b).

Chacun des paramètres de la décision - exigence esthétique du patient, forme des incisives, sensibilité à la carie - devra être évalué et un débat doit être engagé entre le patient, le chirurgien-dentiste traitant et l'orthodontiste.

L'objectif fonctionnel, cinématique, doit prévaloir sur la vision statique. Il est à noter qu'une classe II canine modérée est rarement un obstacle à une protection canine efficace. . 

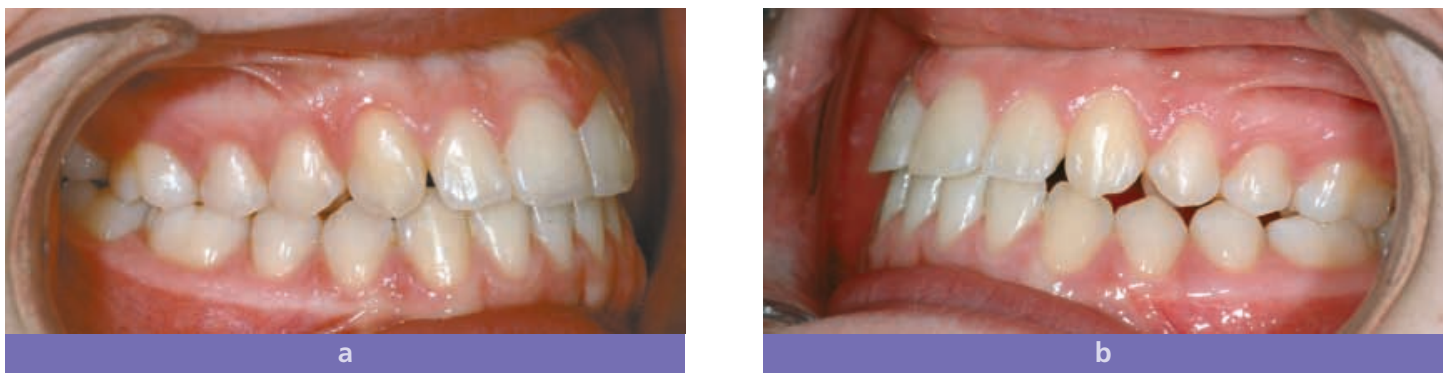

Fig. 4 a et b Dysharmonie dento-dentaire par excès du diamètre mésio-distal des prémolaires maxillaires.

\section{La gestion des agénésies}

Les agénésies les plus fréquentes ayant des répercussions sur la classe I canine sont celles des incisives latérales maxillaires ; mais l'agénésie d'une incisive mandibulaire voire d'une canine n'est pas exceptionnelle.

Le propos de cet article n'est pas de proposer des solutions pour chacune des situations cliniques, tant elles sont nombreuses et la bibliographie pléthorique, mais d'attirer l'attention sur les paramètres de la décision dans le débat «solution prothétique versus fermeture des espaces». Dans ces cas, l'observation, la réflexion, la prise en compte des impératifs de chacun des protagonistes sera plus pertinente que l'édition d'un catalogue dit «de bonne pratique» jamais totalement adapté à la diversité des situations cliniques.
Les impératifs esthétiques, fonctionnels - intégrant la ventilation - de stabilité, le potentiel de croissance et de coopération du patient, les dysmorphoses associées, les impératifs financiers à court ou à long terme et la pérennité des prothèses, doivent être pris en compte (fig. 5 a, b, c). Là encore, il conviendra de se méfier de certains préjugés; la solution implantaire ne sera pas forcément la meilleure sur le plan esthétique en présence d'un sourire gingival découvrant largement les collets des incisives latérales.

En général, la solution prothétique est préférée dans les cas où il existe une dysmorphose associée de type classe III squelettique, avec micro maxillie par déficience sagittale et/ou transversale, associée à une faible perméabilité des voies aérifères supérieures (fig. 6 a à c). II est à
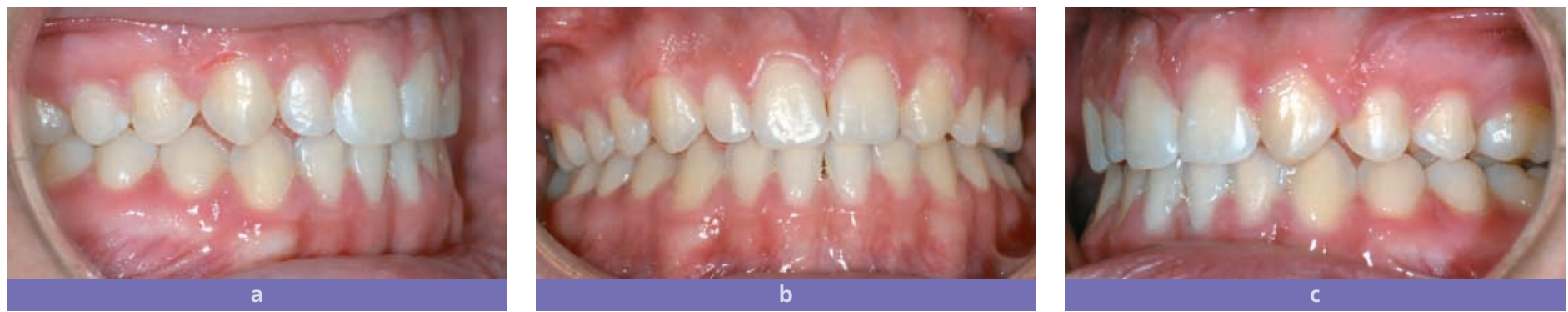

Fig. 5 a à c Agénésie de 22 traitée par fermeture de l'espace. 

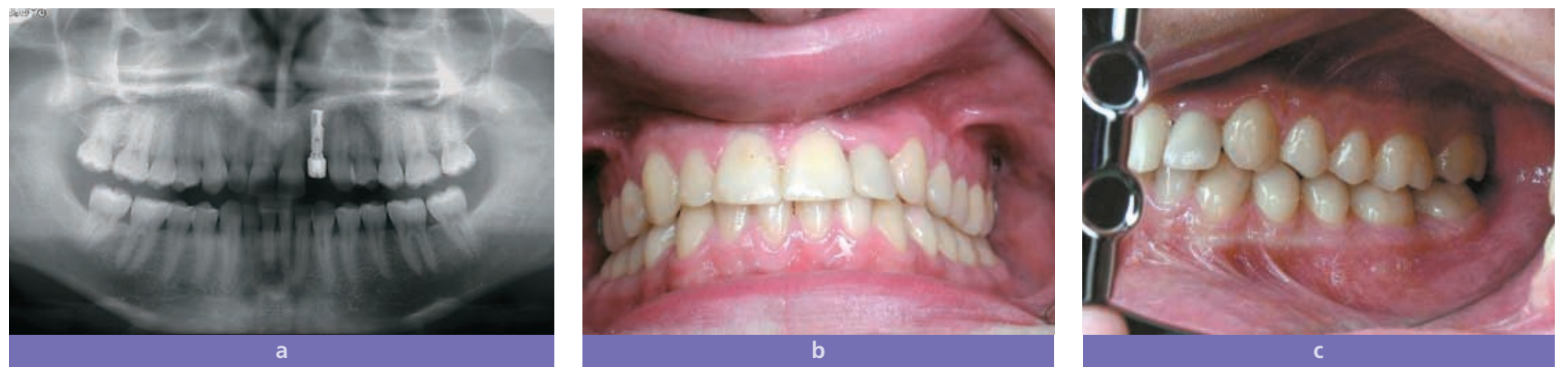

Fig. 6 a à c Agénésie de 22 traitée par ouverture de l'espace et reconstitution prothétique implantoportée.

noter que l'utilisation de mini vis d'ancrage squelettique [12] permettant le mésialage des secteurs latéraux maxillaires sans provoquer le

\section{Les transpositions}

Les plus fréquentes sont la transposition canine/prémolaire et la transposition canine/ incisive latérale.

Leur traitement peut en être très simple - dans le cas d'une transposition canine/ prémolaire associée à une biproalvéolie ou à une dysharmonie dento-maxillaire modérée, l'extraction des prémolaires règle le problème de manière élégante - ou extrêmement complexe, car le croisement de deux dents entre des corticales osseuses, sans provoquer ou recul des incisives ou de réduction du périmètre de l'arcade maxillaire pourrait changer les pratiques.

aggraver des rhizalyses ou des fenestrations osseuses, est assez acrobatique. Elle requiert de la part du chirurgien une bonne technique de dégagement et de collage de la canine et de la part de l'orthodontiste, une bonne maîtrise des mécaniques orthodontiques.

Ces tentatives sont réservées aux cas où la canine est incluse. Après évolution sur l'arcade, la sagesse recommande l'abstention thérapeutique (fig. 7 a à 7 k).
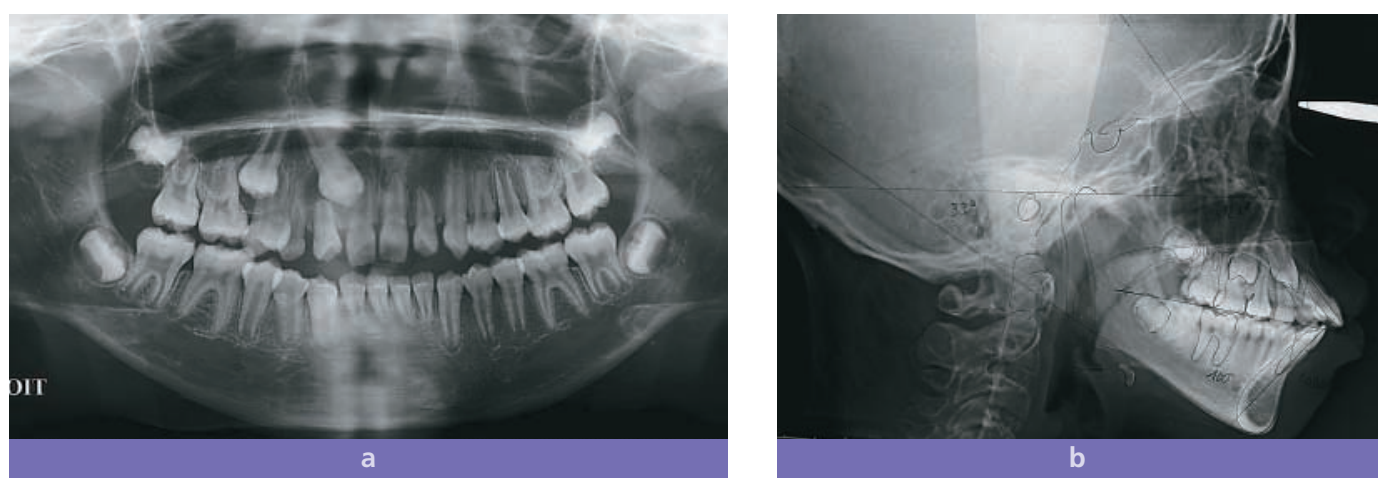

Fig. 7 a et $b$ 

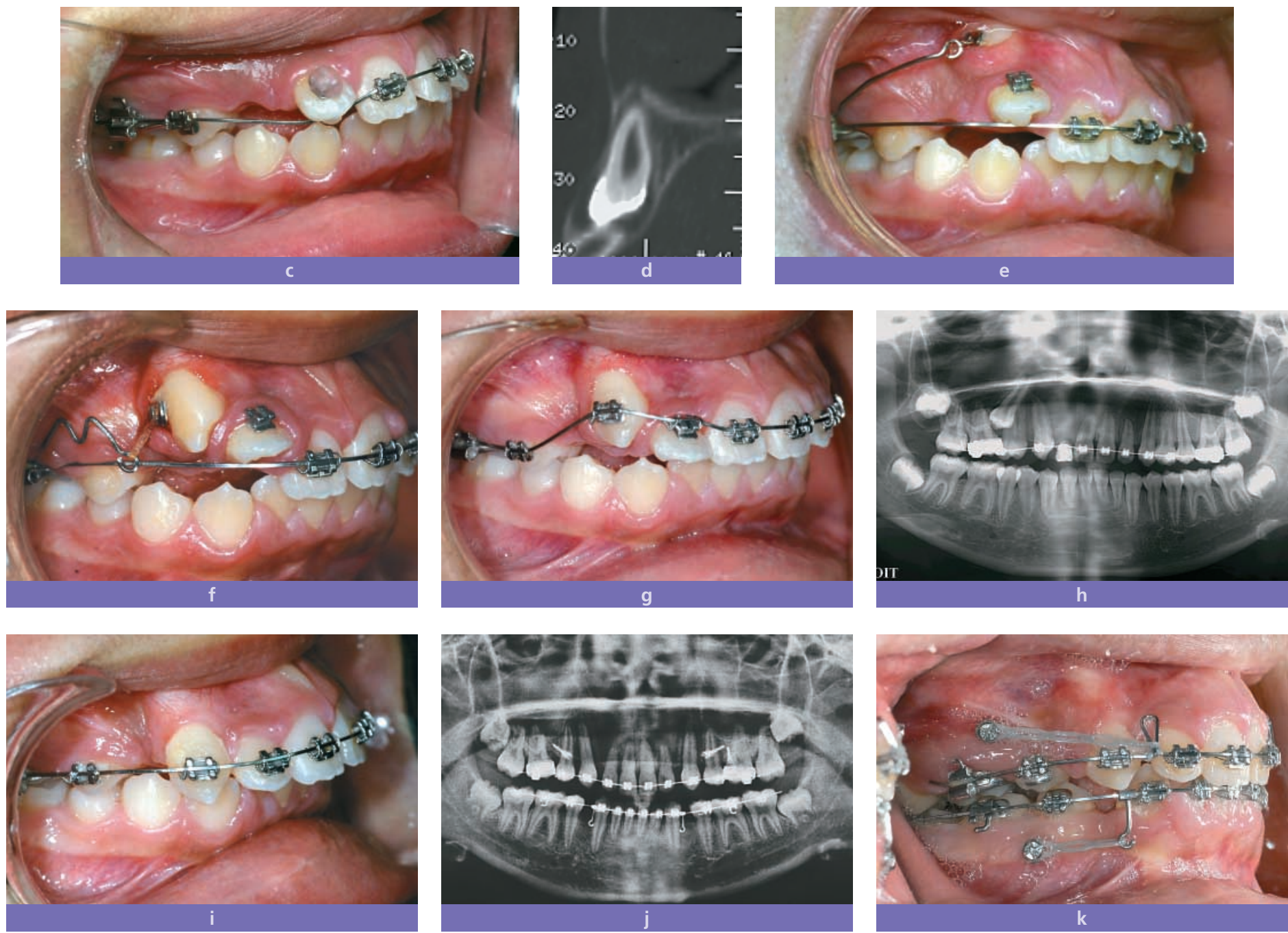

Fig. $7 \mathrm{c}$ à $\mathrm{k}$

Fig. 7 a à $k$ Traitement d'une transposition 13/12 dans un contexte d'inclusions multiples $(13,15)$, de biproalvéolie sévère.

La rhizalyse partielle de 12 et la proximité de la canine avec la racine de 12 sont objectivées par l'examen tomodensitométrique. Un traitement par extraction de 12, 24, 34, 44 avait été envisagé, mais à la demande du chirurgien-dentiste traitant et en accord avec le patient, la correction de la transposition suivie de l'extraction des premières prémolaires a finalement été tenté.

Les figures 7 c et 7 e montrent la version radiculo-palatine de 12, dans le but de réduire le risque d'interférence lors du passage de la canine et d'aggravation de la rhizalyse. Contrairement à l'usage, nous ne recherchons pas un trajet sous-muqueux de 13 pour la même raison. La "disparition» de la racine de 12 sur le cliché panoramique figure 7 h ne doit pas nous inquiéter, la racine n'est plus visible car elle n'est plus dans le plan de coupe de la tomographie. Elle redevient visible après le redressement de 12 , figure $7 \mathrm{j}$ dès que le risque d'interférence est écarté. 


\section{Le maintien d'une biproalvéolie}

Le maintien d'un certain degré de biproalvéolie dans les objectifs de traitement peut se justifier dans les cas où le recul des lèvres dans le profil n'est pas souhaité et dans ceux où les extractions de prémolaires sont contre-indiquées.
La tentative de mise en classe I des canines se traduira par une contrainte occlusale antérieure ou par la persistance de diastèmes antérieurs maxillaires.

\section{Le refus d'un protocole de chirurgie orthognathique}

Le refus d'une étape chirurgicale orthognatique dans le traitement des malocclusions ayant pour origine un décalage squelettique sévère doit nous faire réfléchir aux alternatives thérapeutiques. Nous pouvons comprendre qu'un patient, dûment informé des risques de cette chirurgie, la refuse et nous demande néanmoins si une amélioration de son état est envisageable. Avons-nous le droit de refuser nos soins sous prétexte que nous ne pourrons obtenir la perfection? La récidive, toujours possible après un traitement chirurgico-orthodontique, pourtant conduit dans les règles de

\section{Conclusion}

L'interrogation «La classe I canine est-elle toujours pertinente ?» doit être considérée dans ses deux sens.

Dans son premier sens "La classe I canine estelle encore pertinente ?», nous répondrons sans ambiguïté par l'affirmative.

Les avantages procurés par cette disposition, quand elle peut être atteinte sans autres com- l'art, devrait nous inciter à la plus grande humilité. Gardons aussi à l'esprit que la typologie n'est pas la pathologie; une face longue ou courte, un profil convexe ne doit pas être systématiquement modifié si le patient ne le souhaite pas. Pour autant, nous ne devons pas accéder à toutes ses demandes, notamment purement esthétiques, et nous engager dans des traitements inutiles voire nuisibles pour sa santé [13]. Dans ce domaine, où les certitudes s'estompent, il faut beaucoup de sagesse et $d$ 'humanité pour naviguer entre les compromis sans tomber dans l'écueil de la compromission.

promis, justifient tous nos efforts, la mise en $œ u v r e$ de tous nos moyens. Le soutien de la coopération de notre patient dans le port des appareils auxiliaires n'est pas le moindre de nos efforts.

Être ambitieux pour la qualité des traitements de nos patients est notre responsabilité, notre fierté et notre satisfaction commune. 
Dans son second sens "La classe I canine estelle pertinente pour tous nos patients ?", la réponse est négative.

Quand un compromis est nécessaire, il appartient aux thérapeutes de classer les impératifs, de définir les priorités et d'individualiser les plans de traitement en gardant à l'esprit le rapport bénéfice/contrainte/risque de toute proposition thérapeutique.

Entre ces deux assertions, également justes, qui semblent paradoxales, nous aurons pour guide : notre réflexion, notre liberté thérapeutique, découlant de notre indépendance professionnelle et notre éthique.

Face à une vision technocratique et normative de la médecine qui s'invite dans le débat scientifique, sans toujours respecter la règle du débat contradictoire, nous devons sans cesse rappeler la complexité et la diversité des situa- tions cliniques. Celle-ci, particulièrement en orthopédie dento-faciale, est liée à la fréquence des associations de dysmorphoses et à I'influence de facteurs typologiques dans la décision thérapeutique. Toute standardisation excessive serait une régression des soins et une stérilisation de la pensée.

Le colloque singulier, que nous devons penser pluriel, reste la meilleure garantie du soin le plus adapté à nos patients. Chacun des intervenants : patient, omnipraticien, spécialiste des différentes disciplines, doit pouvoir exposer ses choix, ses priorités, ses limites et ses doutes.

Cette démarche, impérative pour l'orthodontie de l'adulte, doit aussi être la règle dans tous les traitements où un compromis mérite d'être envisagé. Elle replace l'omnipraticien (et son patient) au centre du processus de décision.

\section{Bibliographie}

1. Angle $\mathrm{EH}$.

Malocclusion of the teeth.

$7^{\text {th }}$ ed., Philadelphia:

SSwhite.Dental Mfs Co. 1907.

2. ANAES

(Agence Nationale d'Accréditation et d'Évaluation en Santé).

Recommandation pour

la pratique clinique. Les critères $d$ 'aboutissement du traitement d'orthopédie dento-faciale.

Décembre 2003.

3. URCAM

(Unions Régionales des Caisses d'Assurance Maladie) d'Alsace, Lorraine et Champagne-Ardenne :

Étude des résultats des traitements orthodontiques. 25 juillet 2005.

Cette enquête peut être consultée sur le site :

http://www.urcam. assurance-maladie.frl index.php?id=7810

4. Haïm F., Boury $O$ Étude URCAM ODF. Un danger peut en cacher un autre.

CDF n 1244/45 09/16 février 2006;18-20.

5. Rinchuse DJ. Kandasamy S. Sciote J.
A comtemporary and evidence-based view of canine protected occlusion.

Am J Orthod Dentofacial Orthop 2007;132(1):90-102.

6. Mohlin B, Axelsson $S$, Paulin G, Pietilä T, Bondemark L, Brattström V, Hansen K, Holm AK. TMD in relation to malocclusion and orthodontic treatment. Angle Orthod 2007;77(3): 542-548.

7. Selaimen $\mathrm{CM}$, Jeronymo JC, Brilhante DP, Lima EM, Grossi PK, Grossi ML. 
Occlusal risk factors for temporomandibular disorders.

Angle Orthod 2007;77(3): 471-477.

8. Planas $\mathrm{P}$ La réhabilitation neuro-occlusale. Traduction M. Château. J. Kolf. Masson 1992.

9. Bolton W.A Disharmony in tooth size analysis. Am. J Orthod 1952;48: 504-529.
10. Freeman JE, Maskeroni AJ, Lorton L.

Frequency of Bolton tooth size discrepancy among different malocclusion patients. Am J Orthod 1996;110: 24-27.

11. Oueiss A, Marchal-Sixou $C$, Dallow A, Baron P, Faure $J$.

Dysharmonie dento-dentaire postérieure.
Rev Orthop Dento Faciale 2008;42:9-26.

12. Lee JS, Kim JK, Park Y-C, Vanarsdal JR. Applications cliniques des mini-implants en orthodontie. Traduit par Kattabi S et Liger F. SID éd 2007.

13. Code de la Santé Publique :

Art. R4127-238

(Ancien article 31 du code de déontologie).

\section{SUMMARY}

\section{Is a Class I canine relationship always necessary?}

Frédéric HAÏM

\section{Keywords \\ - objectives of orthodontic treatment \\ - Class I canine relationship \\ - recommendations for conduct of practice \\ - quality of care \\ - interdisciplinary care \\ - compromise}

The attainment of a Class I canine relationship has traditionally been considered a high priority objective in orthodontic treatment. Some studies, ordered by social service authorities, have concluded that its absence at the close of treatment indicates a failure in treatment quality. However in certain clinical situations a Class I canine relationship can be obtained only by adversely affecting other equally important treatment objectives. To assess this dilemma a classification of priorities conducted in a broadly interdisciplinary fashion is indispensable. In view of the diversity of clinical situations orthodontists confront, the limits and dangers of an abusively rigid standardization need to be fully evoked.

Incorporating the wishes of the patient within the framework of individualized treatment planning, inter-disciplinary coordination, reflection, therapeutic freedom, and professional independence in the service of ethical practice, are all measurements of quality health care dedicated to the needs of patients. 